\title{
Vibrationally resolved ionization cross sections for the ground state and electronically excited states of the hydrogen molecule
}

\author{
D. Wünderlich \\ Max-Planck-Institut für Plasmaphysik (IPP), EURATOM Association, Boltzmannstr. 2, \\ D-85748 Garching, Germany
}

\begin{abstract}
Ionization cross sections for the hydrogen molecule have been calculated by applying the classical Gryzinski theory combined with the Franck-Condon theory. It is shown that - if the reaction channels, Franck-Condon factors and Franck-Condon densities are taken into account properly - the calculated cross sections for ionization of the molecular ground state $X^{1} \Sigma_{g}^{+}\left(v^{\prime}=\right.$ $0)$ are in almost perfect agreement with the latest and most reliable experimental results. A comprehensive database is established which includes vibrationally resolved cross sections for non-dissociative and dissociative ionization of the molecular ground state as well as for the first five non-repulsive electronically excited states in the molecule.
\end{abstract}

Keywords: Hydrogen molecule, Ionization, Cross section, Gryzinski theory, Franck-Condon theory

\section{Introduction}

Ionization of the hydrogen molecule and consecutive recombination of the molecular ion play an important role in the kinetics and population dynamics of low-temperature hydrogen plasmas. An example is the divertor plasma in magnetically confined fusion experiments: here detached conditions are preferred in order to reduce the thermal load onto the divertor structure [1, 2]. In these conditions the electron temperature close to the divertor walls is low 
(around a fev eV). Hence the survival length of the molecules produced on the wall and the molecular density in the plasma volume are high. The proton density and consequently proton and energy fluxes are effectively reduced by volume recombination. A volume recombination channel is Molecular Assisted Recombination (MAR) [3, 4] which is based on ionization of hydrogen molecules, followed by dissociative recombination of the molecular ions. It has been shown in [5] that the contribution of MAR to the total plasma recombination in the divertor can be in the order of several $10 \%$

Low-temperature low-pressure hydrogen plasmas, for example in ion sources for neutral beam heating (NBI) of fusion experiments [6], linear plasma generators [7] or small laboratory experiments, can be in the socalled recombining plasma regime, characterized by low electron density and temperature. In such recombining plasmas the recombination of atomic and molecular hydrogen ions is of high relevance for populating excited states in the hydrogen atom. As a result, the line intensity ratios of different spontaneous transitions between electronic states of the atom in recombining plasmas deviates significantly from the line ratios in non-recombining (ionizing) plasmas [8]. This effect can be visualized best in the linear machines where a transition from an ionizing to a recombining plasma is accompanied by a change of the color of the plasma light from red to blue $[9,10]$.

The molecular hydrogen ion $\mathrm{H}_{2}^{+}$is of high relevance for the processes described above. Similar to the hydrogen molecule $\mathrm{H}_{2}$, its ion consists of a ground state and several electronically excited states. All these electronic states contain vibrational and rotational sub-levels. Electronic, vibrational and rotational excitation of the neutral molecule as well as of the ion is of particular importance for the processes described above: the presence of excited states in the molecule can strongly decrease the ionization threshold and thus increase the ionization efficiency. Depending on the plasma parameters, excited states will significantly contribute to the ionization of the molecule. Prominent examples are the vibrational levels in the molecular ground state or the metastable state $c^{3} \Pi_{u}(v=0)$ with relatively high population densities - even at low electron densities. The probability for dissociative recombination of the molecular hydrogen ion strongly depends on the vibrational population of the ion [11]. Concluding, knowledge of accurate vibrationally (and rotationally) resolved ionization and recombination cross sections is essential for modeling low-temperature hydrogen plasmas by using population models, for example Collisional Radiative (CR) $[12,13,14]$ models.

The energetic spacing between rotationally excited levels is small com- 
pared to the electronic states and vibrational levels. Thus, in population models often the very complex structure of the hydrogen molecule is simplified by neglecting the rotational levels. This is done also within this paper which in order to reduce the amount of data.

Only the ground state ${ }^{2} \Sigma_{g}^{+}$of the ion and its first electronically excited state ${ }^{2} \Sigma_{u}^{+}$play a role for ionization of the molecule and recombination of the ion. All other electronic states lie energetically well above these two states [15]. The potential curve of the state ${ }^{2} \Sigma_{g}^{+}$forms a potential well while the state ${ }^{2} \Sigma_{u}^{+}$is repulsive. Figure 1 shows the potential energy curves of the molecular ground state $X^{1} \Sigma_{g}^{+}$and the two considered states in the molecular ion.

Non-dissociative ionization occurs if a neutral molecule is excited from a vibrational level $v^{\prime}$ in an electronic state $p^{\prime}$ into the vibrational level $v^{\prime \prime}$ of the ionic ground state:

$$
\mathrm{H}_{2}\left(p^{\prime}, v^{\prime}\right)+\mathrm{e}^{-} \rightarrow \mathrm{H}_{2}^{+}\left({ }^{2} \Sigma_{g}^{+}, v^{\prime \prime}\right)+2 \mathrm{e}^{-} .
$$

If either the vibrational continuum of the ionic ground state or the repulsive state ${ }^{2} \Sigma_{u}^{+}$is excited, the ion will dissociate into an atom and an atomic ion:

$$
\begin{gathered}
\mathrm{H}_{2}\left(p^{\prime}, v^{\prime}\right)+\mathrm{e}^{-} \rightarrow \mathrm{H}_{2}^{+}\left({ }^{2} \Sigma_{g}^{+}, E^{\prime \prime}\right)+2 \mathrm{e}^{-} \rightarrow \mathrm{H}+\mathrm{H}^{+}+2 \mathrm{e}^{-}, \\
\mathrm{H}_{2}\left(p^{\prime}, v^{\prime}\right)+\mathrm{e}^{-} \rightarrow \mathrm{H}_{2}^{+}\left({ }^{2} \Sigma_{u}^{+}, E^{\prime \prime}\right)+2 \mathrm{e}^{-} \rightarrow \mathrm{H}+\mathrm{H}^{+}+2 \mathrm{e}^{-},
\end{gathered}
$$

where $E^{\prime \prime}$ is the excitation energy.

Reactions (1) and (2) consist of removing one of the two orbital electrons (which are both initially in the state $1 \sigma_{g}$ ) from the molecule accompanied by vibrationally exciting the ion. During reaction (3) an additional reaction step takes place: the second orbital electron is excited into the state $1 \sigma_{u}$ [16].

In figure 1 the vibrational Eigenfunctions of $v^{\prime}=0$ in the molecular ground state and $v^{\prime \prime}=0$ in the ionic ground state are shown as well as two wave functions of free particles connecting to the vibrational continuum of the ionic ground state and the repulsive state. The three vertical arrows represent the ionizing processes (1)-(3).

The accuracy of theoretical cross sections for processes (1)-(3) strongly depends on the treatment of the different reaction channels. Up to now no complete (i.e. accounting for the vibrational levels $v^{\prime}$ and $v^{\prime \prime}$ in several electronic states of the molecule and the three processes (1)-(3)) and consistent 


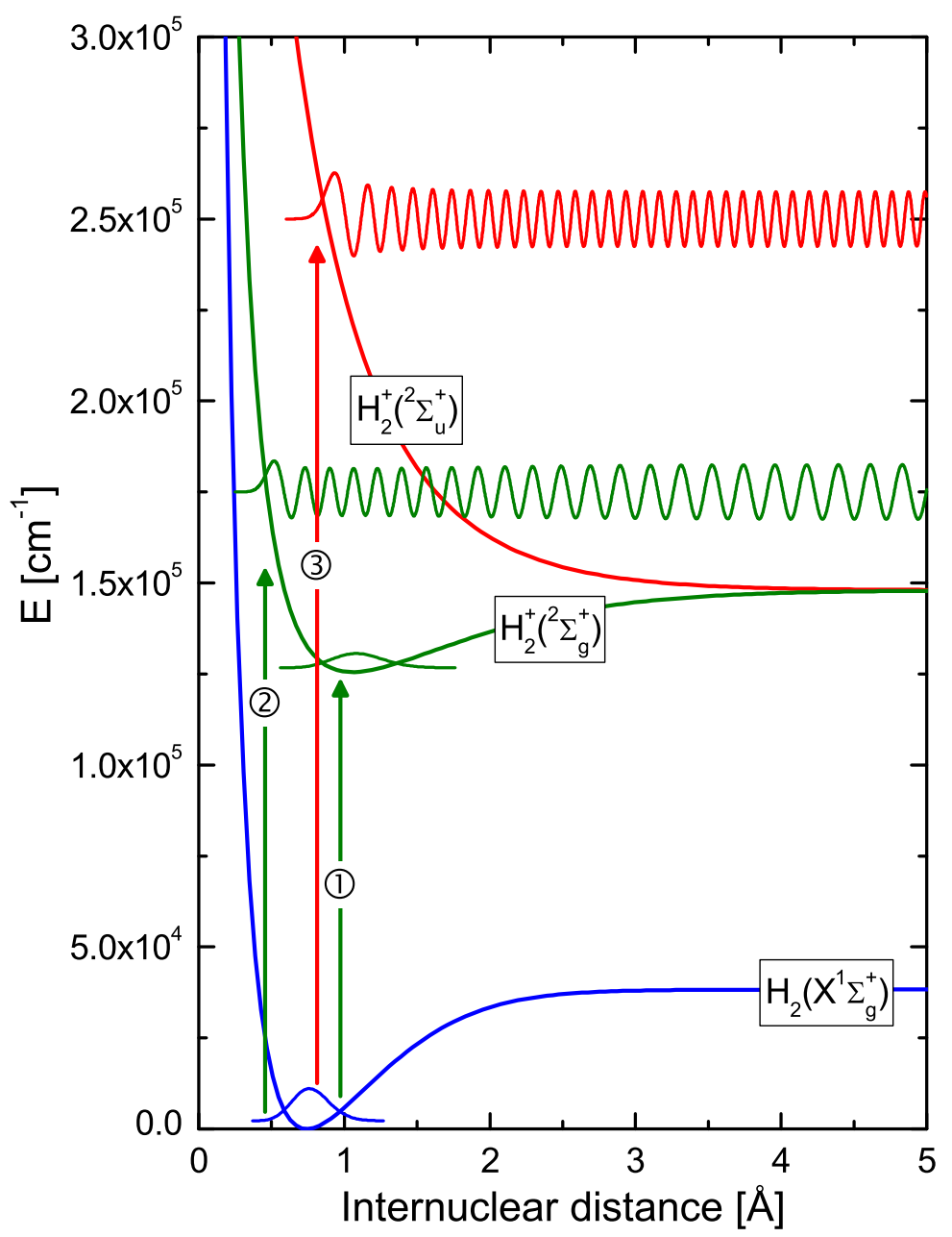

Figure 1: Potential energy curves for the ground state of the hydrogen molecule and the first two electronically excited state in the ion. Eigenfunctions of $v^{\prime}=0$ in the molecular ground state, $v^{\prime \prime}$ in the ionic ground state and wave functions of two free particles. Indicated by vertical arrows are the non-dissociative ionization channel (1) and the two dissociative channels (2) and (3). 
data set is available in literature. Some efforts has been done in the past $[17,18,19]$, but these results are based on a simplified framework: the excitation of the second orbital electron into the state $1 \sigma_{u}$ taking place during reaction (3) was neglected and/or the Franck-Condon densities needed to describe the excitation into the vibrational continuum have been calculated by means of simple approximations.

Aim of this paper is to establish a comprehensive and accurate database containing vibrationally resolved cross sections for non-dissociative and dissociative ionization of the hydrogen molecule. The paper first gives an introduction about the evaluation of Franck-Condon factors and Franck-Condon densities. Then, the Gryzinski framework for calculating electron collision cross sections is briefly described and linked to the Franck-Condon theory in order to obtain vibrationally resolved results. The applicability of the Gryzinski method is verified by comparing the calculated ionization cross sections for the level $v^{\prime}=0$ in the molecular ground state with available experimental and theoretical data. Finally, the vibrationally resolved cross sections for non-dissociative and dissociative ionization of the electronic states $X^{1} \Sigma_{g}^{+}$, $E F^{1} \Sigma_{g}^{+}, B^{1} \Sigma_{u}^{+}, C^{1} \Pi_{u}, a^{3} \Sigma_{g}^{+}$and $c^{3} \Pi_{u}$ in the molecule are presented.

\section{Computational method}

\subsection{Franck-Condon factors and Franck-Condon densities}

Franck-Condon factors (FCF) and Franck-Condon densities (FCD) describe the overlap of two vibrational wave functions in a molecule [20,21]. In the Franck-Condon approximation, the internuclear distance is considered to be fixed during an electronic transition. If the perturbing Hamiltonian of the transition does not depend on the internuclear distance, then according to Fermi's Golden Rule the FCF or FCD quantify the transition probability from one wave function to another.

The Franck Condon factor $q_{v^{\prime} v^{\prime \prime}}^{p^{\prime \prime}}$ for a transition between the vibrational level $v^{\prime}$ in the electronically excited state $p^{\prime}$ and the vibrational level $v^{\prime \prime}$ in the electronic state $p^{\prime \prime}$ of a diatomic molecule can be calculated by a simple convolution of the vibrational Eigenfunctions:

$$
q_{v^{\prime} v^{\prime \prime}}^{p^{\prime} p^{\prime \prime}}=\left|\int \psi_{v^{\prime}}^{p^{\prime}}(r) \psi_{v^{\prime \prime}}^{p^{\prime \prime}}(r) \mathrm{d} r\right|^{2} .
$$


If either $p^{\prime \prime}$ is repulsive or the transition ends in the vibrational continuum of $p^{\prime \prime}$, the discrete FCF split up into the continuous FCD $Q_{v^{\prime} E^{\prime \prime}}^{p^{\prime \prime}}$ :

$$
Q_{v^{\prime} E^{\prime \prime}}^{p^{\prime} p^{\prime \prime}} \mathrm{d} E^{\prime \prime}=\frac{2}{h} \sqrt{\frac{2 \mu}{\left(E^{\prime \prime}-E_{\text {diss }}^{p^{\prime \prime}}\right)}}\left|\int \psi_{v^{\prime}}^{p^{\prime}}(r) \psi_{E^{\prime \prime}}^{p^{\prime \prime}}(r) \mathrm{d} r\right|^{2} \mathrm{~d} E^{\prime \prime} .
$$

The wave function $\psi_{E^{\prime \prime}}^{p^{\prime \prime}}$ of the free particle has to be normed to an amplitude of one in the outbound region. $E^{\prime \prime}$ is the excitation energy, $E_{\text {diss }}^{p^{\prime \prime}}$ the dissociation energy of the electronic state $p^{\prime \prime}$ and $\mu$ the reduced mass of the molecule.

Since the calculation of FCD is numerically more demanding than the calculation of FCF, often the $\delta$-function approximation is applied [18]. In this approximation the overlap integral of the vibrational Eigenfunction $\psi_{v^{\prime}}^{p^{\prime}}$ and the wave function $\psi_{E^{\prime \prime}}^{p^{\prime \prime}}$ is reduced to the product of both functions at the classical turning point $r_{\mathrm{tp}}^{p^{\prime \prime}}$ of $\psi_{E^{\prime \prime}}^{p^{\prime \prime}}$ at the energy $E^{\prime \prime}$. As additional simplification, the value of $\psi_{E^{\prime \prime}}^{p^{\prime \prime}}$ at this internuclear distance is approximated by the inverse derivation of the potential energy curve $U^{p^{\prime \prime}}$ :

$$
Q_{v^{\prime} E^{\prime \prime}}^{p^{\prime} p^{\prime \prime}} \mathrm{d} E^{\prime \prime}=\left.\left|\left(\frac{\mathrm{d} U^{p^{\prime \prime}}(r)}{\mathrm{d} r}\right)_{r=r_{\mathrm{tp}}^{p^{\prime \prime}}\left(E^{\prime \prime}\right)}^{-1}\right| \psi_{v^{\prime}}^{p^{\prime}}(r)\right|_{r=r_{\mathrm{tp}}^{p^{\prime \prime}}\left(E^{\prime \prime}\right)} ^{2} \mid \mathrm{d} E^{\prime \prime} .
$$

In general, the following closure relation is satisfied:

$$
\sum_{v^{\prime \prime}} q_{v^{\prime} v^{\prime \prime}}^{p^{\prime} p^{\prime \prime}}+\int_{E^{\prime \prime}} Q_{v^{\prime} E^{\prime \prime}}^{p^{\prime} p^{\prime \prime}} \mathrm{d} E^{\prime \prime}=1
$$

The relative contributions of excitation into bound vibrational states and dissociative excitation into the vibrational continuum depend on the shape of the potential curves. In case of a repulsive upper state $p^{\prime \prime}$ no bound excitation is possible and the first part of the left hand side in equation (7) yields zero.

In order to calculate non-dissociative and dissociative ionization cross sections for the hydrogen molecule, the corresponding FCF and FCD have been calculated using the computer code TraDiMo [22]. This code determines the Eigenfunctions and wave functions by solving the Schrödinger equation:

$$
\left(-\frac{\hbar}{2 \mu} \frac{\partial^{2}}{\partial r^{2}}+U^{p}(r)\right) \psi_{v}^{p}(r)=E_{v}^{p} \psi_{v}^{p}(r),
$$


where $E_{v}^{p}$ is the Eigenvalue for the vibrational level $v$ in the electronic state $p$ and $\psi_{v}^{p}(r)$ the corresponding wave function. $U^{p}$ is the potential energy curve of $p$. This second-order differential equation is transformed into two first-order ordinary differential equations which are then solved numerically using the Taylor progression.

While a complete set of FCF for ionizing processes in the hydrogen molecule and its isotopomeres has been published elsewhere [23], some of the FCD calculated within the scope of this paper are presented in section 3 .

\subsection{Ionization cross sections}

Several quantum mechanical and classical techniques are available to calculate cross sections for molecular excitation and ionization processes. In principle, quantum mechanical calculations are more sophisticated and can be assumed to be more accurate than the classical approach. Nevertheless, due to the amount of cross sections to be calculated, a semi-classical modification of the classical Gryzinski method was applied within the scope of this paper.

The classical framework developed by Gryzinski [24, 25] and rephrased by Bauer [25] provides a set of simple equations which describe cross sections for different kinds of energy transfer to atoms or molecules. In principle, these equations are based on the classical model by Thomson [26]. The main difference is that within the Thomson theory the kinetic energy of orbital electrons is set to zero. In contrast, the Gryzinski theory accounts for the non-zero kinetic energy and the energy distribution of the electrons.

One of the most essential equations of the Gryzinski theory describes the cross section for the process in which an orbital electron (all other orbital electrons are unaffected) gains energy by electron collision. Most other equations of the Gryzinski framework can be deduced from this equation:

$$
\begin{gathered}
\sigma_{E_{\mathrm{thr}}}^{\text {gain }}\left(E_{\mathrm{e}}\right)=\frac{N_{\mathrm{e}} \sigma_{0}}{E_{\mathrm{thr}}^{2}} \sqrt{\frac{\varepsilon^{2} E_{\mathrm{e}}}{\left(\varepsilon+E_{\mathrm{e}}\right)^{3}}}\left(1-\frac{E_{\mathrm{thr}}}{E_{\mathrm{e}}}\right)^{\frac{2 \varepsilon+E_{\mathrm{thr}}}{\varepsilon+E_{\mathrm{thr}}}} \\
\cdot\left\{\frac{E_{\mathrm{thr}}}{\varepsilon}+\frac{2}{3}\left[1-\frac{E_{\mathrm{thr}}}{2 E_{\mathrm{e}}}\right] \ln \left(\exp (1)+\sqrt{\frac{E_{\mathrm{e}}-E_{\mathrm{thr}}}{\varepsilon}}\right)\right\},
\end{gathered}
$$

where $E_{\mathrm{e}}$ is the initial energy of the impinging electron and $\varepsilon$ the initial kinetic energy of the orbital electron to be excited. $N_{\mathrm{e}}$ is the effective number of 
equivalent electrons in the initial state of the transition and can be set to two and one in case of the ground state and electronically excited states of the hydrogen molecule, respectively. $\sigma_{0}$ is equal to $6.56 \cdot 10^{-18} \mathrm{~m}^{2} \mathrm{eV}^{2}$ [24] and $E_{\text {thr }}$ is the lower limit of the energy gain (i.e. the threshold energy).

In equation (9) no upper limit of the energy gain is defined. Such a limit has to be considered in case of excitation of specific electronically (and/or vibrationally or rotationally in a molecule) excited states. For energies above a certain limit the excitation by electron collision no longer solely populates the designated state but also one or more of the energetically higher lying states. The cross section for an energy gain constricted by an upper limit can be approximated by applying equation (9) once for the threshold energy and the upper energy limit and subtracting the results. If more than one state is available for excitation in a small energy range, the effective number of electrons $N_{\mathrm{e}}$ need to be reduced in an appropriate manner [25].

Usually, the initial kinetic energy $\varepsilon$ of the orbital electron can be set to the ionization potential $I$ of the atom or molecule [25]. The ionization potential is equal to the lowest threshold energy of all ionizing processes possible. If the process for which the cross section is to be calculated has a threshold equal the ionization potential, also $E_{\text {thr }}$ can be set to $I$. Then equation (9) reduces to the well known Gryzinski ionization cross section:

$$
\begin{array}{r}
\sigma^{\text {ion }}\left(E_{\mathrm{e}}\right)=\frac{N_{\mathrm{e}} \sigma_{0}}{E_{\mathrm{e}} I}\left(\frac{\left(E_{\mathrm{e}} / I\right)-1}{\left(E_{\mathrm{e}} / I\right)+1}\right)^{3 / 2} \\
\cdot\left[1+\frac{2}{3}\left(1-\frac{I}{2 E_{\mathrm{e}}}\right) \ln \left(\exp (1)+\sqrt{\frac{E_{\mathrm{e}}}{I}-1}\right)\right] .
\end{array}
$$

This equation must not be used for ionizing processes with threshold energy larger than the ionization potential. This is the case - for example - when the ionization produces an excited level of the ion. Then equation (9) has to be applied.

As mentioned before, reactions (1) and (2) consist of removing one of the orbital electrons from the molecule accompanied by vibrationally exciting the ion. In order to easily obtain vibrationally resolved ionization cross sections for these reactions, the Gryzinski theory can be combined with the FranckCondon theory. The cross section for a non-dissociative ionizing electron collision from the vibrational level $v^{\prime}$ within the electronic level $p^{\prime}$ of the molecule to the vibrational level $v^{\prime \prime}$ in the bound state $p^{\prime \prime}$ of the ion can be 
calculated as follows:

$$
\sigma_{\text {ndiss }}^{\text {ion }}\left(E_{\mathrm{e}}, p^{\prime}, v^{\prime}, p^{\prime \prime}, v^{\prime \prime}\right)=q_{v^{\prime} v^{\prime \prime}}^{p^{\prime} p^{\prime \prime}} \cdot \sigma_{E_{\mathrm{thr}}}^{\text {gain }}\left(E_{\mathrm{e}}\right),
$$

with $E_{\text {thr }}$ equal to the threshold energy of the considered ionizing reaction. If information on the final vibrational state $v^{\prime \prime}$ is not required, the corresponding cross sections can be added together in order to obtain a total ionization cross section for the vibrational state $v^{\prime}$ :

$$
\sigma_{\text {ndiss }}^{\text {ion }}\left(E_{\mathrm{e}}, p^{\prime}, v^{\prime}, p^{\prime \prime}\right)=\sum_{v^{\prime \prime}} q_{v^{\prime} v^{\prime \prime}}^{p^{\prime} p^{\prime \prime}} \cdot \sigma_{E_{\text {thr }}}^{\text {gain }}\left(E_{\mathrm{e}}\right)
$$

The dissociative ionization by excitation into the vibrational continuum of the ionic ground state ${ }^{2} \Sigma_{g}^{+}$can be treated in a similar manner:

$$
\sigma_{\text {diss }}^{\text {ion }}\left(E_{\mathrm{e}}, p^{\prime}, v^{\prime}, p^{\prime \prime}\right)=\int_{E^{\prime \prime}}^{E_{\mathrm{e}}} Q_{v^{\prime} E^{\prime \prime}}^{p^{\prime} p^{\prime \prime}} \cdot \sigma_{E^{\prime \prime}}^{\text {gain }}\left(E_{\mathrm{e}}\right) \mathrm{d} E^{\prime \prime}
$$

If the dissociative ionization occurs via the repulsive state ${ }^{2} \Sigma_{u}^{+}$of the ion (equation (3)), additionally the remaining orbital electron has to be excited from the state $1 \sigma_{g}$ into the state $1 \sigma_{u}$. The resulting two-step reaction can by treated by similar means as double ionization. The evaluation of cross sections for double ionization using the Gryzinski theory is described in detail in $[24,16]$. Due to the energy dependence of the specific involved cross sections it is possible to reduce several nested integrals and obtain simple equations for the cross section for two different reaction channels: either the incident electron removes one orbital electron from the molecule and then excites the second orbital electron to $1 \sigma_{u}$ (channel (a)). Or the incident electron removes one orbital electron from the molecule and then this orbital electron excites the second orbital electron to $1 \sigma_{u}$ (channel (b)). The cross sections of channel (a) and (b) can be written as:

$$
\begin{gathered}
\sigma_{\text {diss }, \mathrm{a}}^{\text {ion }}\left(E_{\mathrm{e}}, E^{\prime \prime}\right)=\frac{N_{\mathrm{e}}}{A}\left(\sigma_{E^{\prime \prime}}^{\text {gain }}\left(E_{\mathrm{e}}\right)-\sigma_{E_{\mathrm{e}}-E_{\mathrm{thr} 2}}^{\text {gain }}\left(E_{\mathrm{e}}\right)\right) \\
\cdot \sigma_{E_{\mathrm{thr} 2}}^{\text {gain }}\left(E_{\mathrm{e}}-<\Delta E_{a}>\right), \\
\sigma_{\text {diss }, \mathrm{b}}^{\text {ion }}\left(E_{\mathrm{e}}, E^{\prime \prime}\right)=\frac{N_{\mathrm{e}}}{A} \sigma_{E^{\prime \prime}+E_{\mathrm{thr} 2}}^{\text {gain }}\left(E_{\mathrm{e}}\right) \cdot \sigma_{E_{\mathrm{thr} 2}}^{\text {gain }}\left(<\Delta E_{b}>\right) .
\end{gathered}
$$

where $A$ is the effective area of the cloud of orbital electrons and $E_{\text {thr2 }}$ the threshold for the excitation of the remaining orbital electron. In [16] values 
for $A$ and $E_{\text {thr2 }}$ are given: for the molecular ion the area $A$ is equal to $6.90 \cdot 10^{-20} \mathrm{~m}^{2}$. A threshold energy of $12.4 \mathrm{eV}$ produces protons with an energy of $2.5 \mathrm{eV}$. In equations (14) and (15) the threshold for excitation of the electron into the orbital $1 \sigma_{u}$ is needed - i.e. the production of protons with zero energy. The possible additional energy gain of the produced proton is accounted for by multiplying with the Franck-Condon density. Thus, for the calculations $E_{\text {thr2 }}$ is set to $9.9 \mathrm{eV} .\left\langle\Delta E_{a}\right\rangle$ is the average energy loss of the incident electron after the first collision of reaction channel (a) and $<\Delta E_{b}>$ is the average energy gain of the removed orbital electron after the first collision of reaction channel (b). These average energies can be calculated as follows:

$$
\begin{array}{r}
<\Delta E_{a}>=\frac{\ln \left(\left(E_{\mathrm{e}}-E_{\mathrm{thr} 2}\right) / E_{\mathrm{thr} 2}\right)}{1-E^{\prime \prime} /\left(E_{\mathrm{e}}-E_{\mathrm{thr} 2}\right)}, \\
<\Delta E_{b}>=\left(E^{\prime \prime}+E_{\mathrm{thr} 2}\right) \cdot \frac{\ln \left(E_{\mathrm{e}} /\left(E^{\prime \prime}+E_{\mathrm{thr} 2}\right)\right)}{1-\left(E^{\prime \prime}+E_{\mathrm{thr} 2}\right) / E_{\mathrm{e}}} .
\end{array}
$$

For electron energies close to the threshold energy $E_{\text {thr2 }}$ the value of $<$ $\Delta E_{a}>$ can be negative. In order to avoid non-physical results, in this case the negative value is replaced by zero during the calculation. The two cross sections for channels (a) and (b) are added together and integrated — similar to equation (13):

$$
\sigma_{\text {diss }}^{\text {ion }}\left(E_{\mathrm{e}}, p^{\prime}, v^{\prime}, p^{\prime \prime}\right)=\int_{E^{\prime \prime}+E_{\mathrm{thr} 2}}^{E_{\mathrm{e}}} Q_{v^{\prime} E^{\prime \prime}}^{p^{\prime} p^{\prime \prime}} \cdot\left(\sigma_{\text {diss } \mathrm{a}}^{\text {ion }}\left(E_{\mathrm{e}}, E^{\prime \prime}\right)+\sigma_{\text {diss }, \mathrm{b}}^{\text {ion }}\left(E_{\mathrm{e}}, E^{\prime \prime}\right)\right) \mathrm{d} E^{\prime \prime},
$$

resulting in the cross section for dissociative ionization of the vibrational level $v^{\prime}$ in the electronic state $p^{\prime}$ of the hydrogen molecule via the repulsive state ${ }^{2} \Sigma_{u}^{+}$.

\section{Results and discussion}

\subsection{Franck-Condon densities}

FCD have been calculated using TraDiMo for the transitions from the molecular states $X^{1} \Sigma_{g}^{+}, E F^{1} \Sigma_{g}^{+}, B^{1} \Sigma_{u}^{+}, C^{1} \Pi_{u}, a^{3} \Sigma_{g}^{+}$and $c^{3} \Pi_{u}$ into the vibrational continuum of the ionic ground state ${ }^{2} \Sigma_{g}^{+}$and into the repulsive state ${ }^{2} \Sigma_{u}^{+}$. As shown in section 2.2, these FCD are a prerequisite for the calculation of cross sections for the corresponding transitions. The potential 
Table 1: Check of the closure relation for Franck-Condon factors and Franck-Condon densities. The table contains values for the transition $H_{2}\left(X^{1} \Sigma_{g}^{+}, v^{\prime}\right) \rightarrow H_{2}^{+}\left({ }^{2} \Sigma_{g}^{+}, v^{\prime \prime}\right)$.

\begin{tabular}{rccc}
\hline $\mathrm{v}^{\prime}$ & $\sum_{v^{\prime \prime}} \mathrm{FCF}$ & $\int_{E^{\prime \prime}} \mathrm{FCD}$ & $\sum_{v^{\prime \prime}}+\int_{E^{\prime \prime}}$ \\
\hline 0 & 0.9852 & 0.0148 & 1.0000 \\
1 & 0.9130 & 0.0870 & 0.9999 \\
2 & 0.7828 & 0.2171 & 0.9999 \\
3 & 0.6986 & 0.3014 & 1.0000 \\
4 & 0.7163 & 0.2837 & 1.0000 \\
5 & 0.7331 & 0.2668 & 1.0000 \\
6 & 0.6991 & 0.3009 & 0.9999 \\
7 & 0.6968 & 0.3031 & 1.0000 \\
8 & 0.7300 & 0.2700 & 1.0000 \\
9 & 0.7359 & 0.2641 & 0.9999 \\
10 & 0.7377 & 0.2623 & 1.0000 \\
11 & 0.7732 & 0.2268 & 1.0000 \\
12 & 0.8240 & 0.1760 & 1.0000 \\
13 & 0.8763 & 0.1237 & 1.0000 \\
14 & 0.9417 & 0.0581 & 0.9998 \\
\hline
\end{tabular}

curves of the six molecular states have been taken from [27], [28, 29], [30], [31], $[32,33]$ and $[32,34]$, respectively. The potential curves of the ionic curves are from [15] and [35]. To qualify the potential curves for the calculation of FCD, the fitting procedure described in [22] has been performed.

In order to estimate the accuracy of the FCD, the closure relation (7) was checked for ionization of the five considered molecular states. The FCF were taken from [23]. As example, the result for transition of the molecular ground state into the ionic ground state ${ }^{2} \Sigma_{g}^{+}$is shown in table 1 . The closure relation if fulfilled almost perfectly. Small deviations (around 0.01\%) from the expected sum one occur for some vibrational levels and are caused by the numeric solution procedure. For the highest vibrational quantum number $v^{\prime}=14$ a slightly higher deviation from one (around $0.02 \%$ ) is observed. Since the Eigenvalue of this vibrational state is very close to the dissociation energy, the Eigenfunction reacts very sensitively on small numerical errors of the calculated Eigenvalue. The closure relation is fulfilled very well also for the molecular states and ionization channels not shown here.

The correspondence principle states that in the limit of very high quan- 


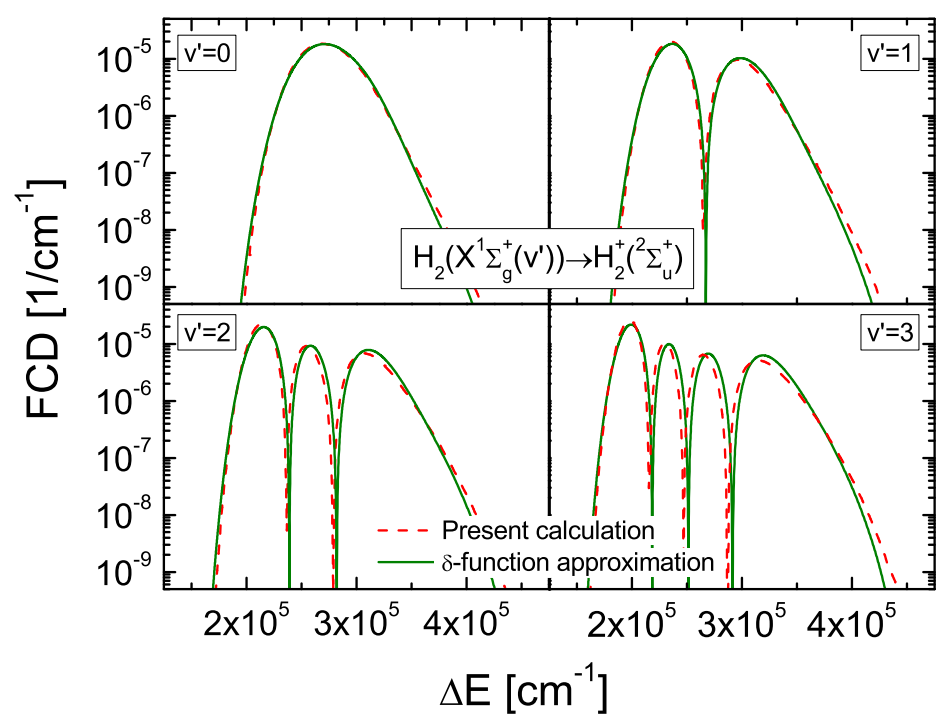

Figure 2: Franck-Condon densities for excitation from the first four vibrational levels in the molecular ground state into the repulsive ${ }^{2} \Sigma_{u}^{+}$state in the ion. Compared are results of the present calculation and the $\delta$-function approximation.

tum numbers the behavior predicted by quantum mechanics reproduces the classical physics. For example, in the case of very high vibrational quantum numbers the probability to find the system close to the classical turning points is higher than the probability to find it somewhere else. Consequently, the vertical transition at the internuclear distance equal to the classical turning point $r_{\text {tp }}^{p^{\prime \prime}}$ can contribute significantly to the absolute value of FCD for dissociative ionization. This fact is utilized - as described earlier — by the $\delta$-function approximation.

During dissociative ionization via the repulsive state ${ }^{2} \Sigma_{u}^{+}$the position of the classical turning point can in principle correspond to any position above the potential well of the initial state of the transition. Thus, the shape of FCD for transitions ending in this state roughly reproduces the shape of the square of the vibrational Eigenfunction of the initial level. This can be seen in figure 2 where the FCD calculated with TraDiMo for the transition from the first four vibrational levels in the molecular ground state to ${ }^{2} \Sigma_{u}^{+}$are shown. For comparison, results of the $\delta$-function approximation are plotted as well. The results of the approximation agree very good with the present results: deviations between the two calculation methods are small for large values of 


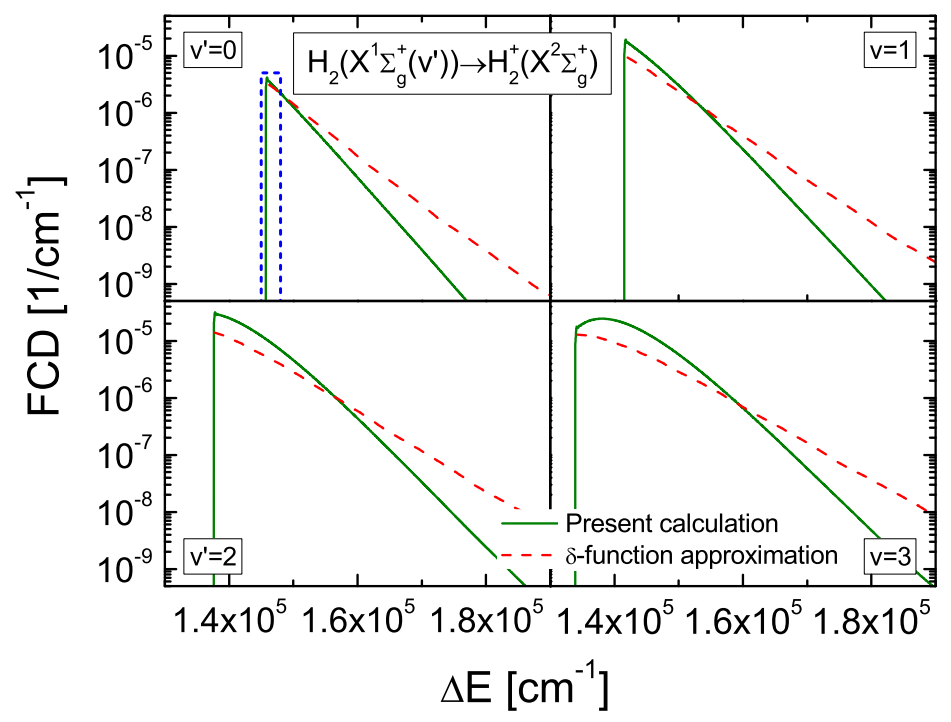

Figure 3: Franck-Condon densities for excitation from the first four vibrational levels in the molecular ground state into the vibrational continuum of the ${ }^{2} \Sigma_{g}^{+}$state in the ion. Compared are results of the present calculation and the $\delta$-function approximation.

the FCD and increase to a significant value only for energies at which the FCD are more than two orders of magnitude smaller than their maximum.

The picture changes drastically when transitions into the vibrational continuum of the ionic ground state ${ }^{2} \Sigma_{g}^{+}$are examined. The internuclear distance of the relevant classical turning points $r_{\mathrm{tp}}^{p^{\prime \prime}}$ in ${ }^{2} \Sigma_{g}^{+}$is smaller than $0.6 \AA$. This is close to the leftmost classical turning points of the states in the neutral molecule. This means that evaluation of the $\delta$-function approximation neglects the region around the maximum of the vibrational Eigenfunctions in the molecule, resulting in a large possible errors. This fact is illustrated in figure 3 where FCD calculated with TraDiMo for ionization of the molecular ground state into the ionic ground state are compared with the results of the approximation. Significant deviations are observed even for high values of the FCD. According to this, dissociative ionization cross sections based on FCD calculated using the $\delta$-function approximation will have a high uncertainty.

Additionally, as a matter of principle, the $\delta$-function approximation can not describe correctly the behavior of FCD very close to the dissociation energy of non-repulsive states, i.e. the energy region in which a successive transition between bound vibrational states and the vibrational continuum 


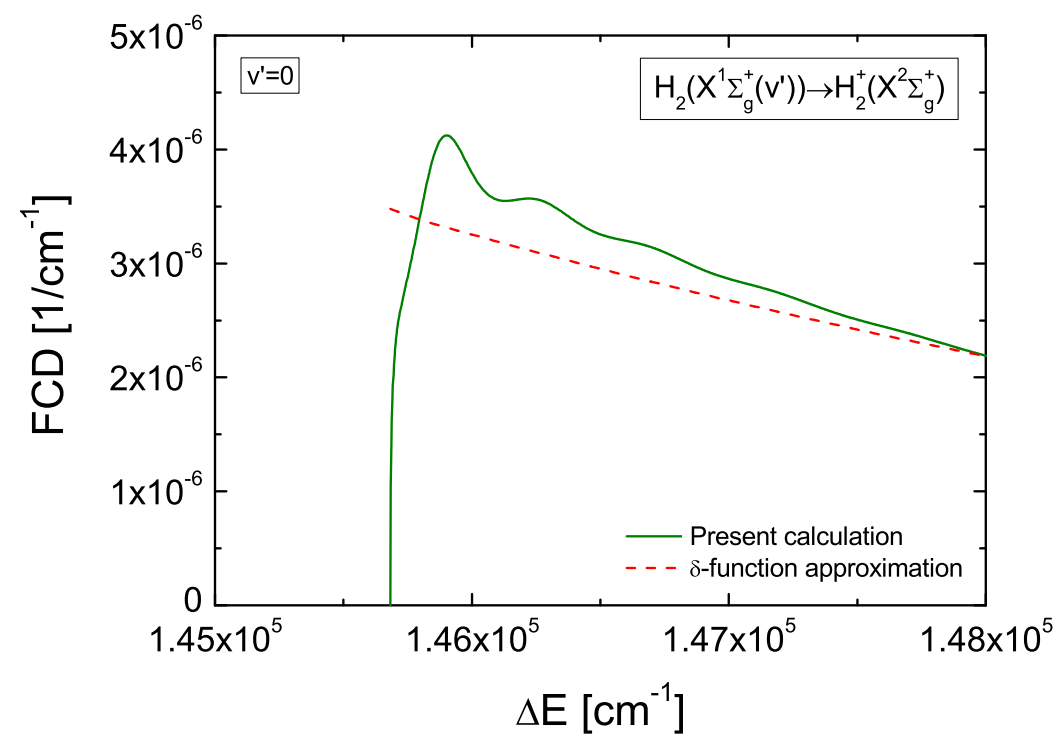

Figure 4: Threshold region of the Franck-Condon densitiy for excitation from $v^{\prime}=0$ in the molecular ground state into the vibrational continuum of the ${ }^{2} \Sigma_{g}^{+}$state in the ion. Compared are results of the present calculation and the $\delta$-function approximation.

takes place. Here, the wave functions of free particles are strongly influenced by the presence of the subjacent potential well. As a result, close to the threshold energy for dissociative ionization the FCD show a oscillating behavior, while FCD determined using the approximation follows a straight line. This can be seen in figure 4, showing a zoom into the left upper part $\left(v^{\prime}=0\right)$ of figure 3 . Concluding, it is not appropriate to apply the simple $\delta$-function approximation and an more exact calculation has to be performed instead - at least for the transitions into the vibrational continuum of the ionic ground state ${ }^{2} \Sigma_{g}^{+}$.

\subsection{Ionization cross sections}

To verify the applied calculation methods, the ionization cross sections are compared with experimental and theoretical results. Only data for $v^{\prime}=0$ in the molecular ground state $X^{1} \Sigma_{g}^{+}$are available in literature.

Figure 5 shows this comparison for non-dissociative ionization. The present cross section (continuous line) is in very good agreement with the experimental data $[36,37,38,39]$. The only theoretical data set available 


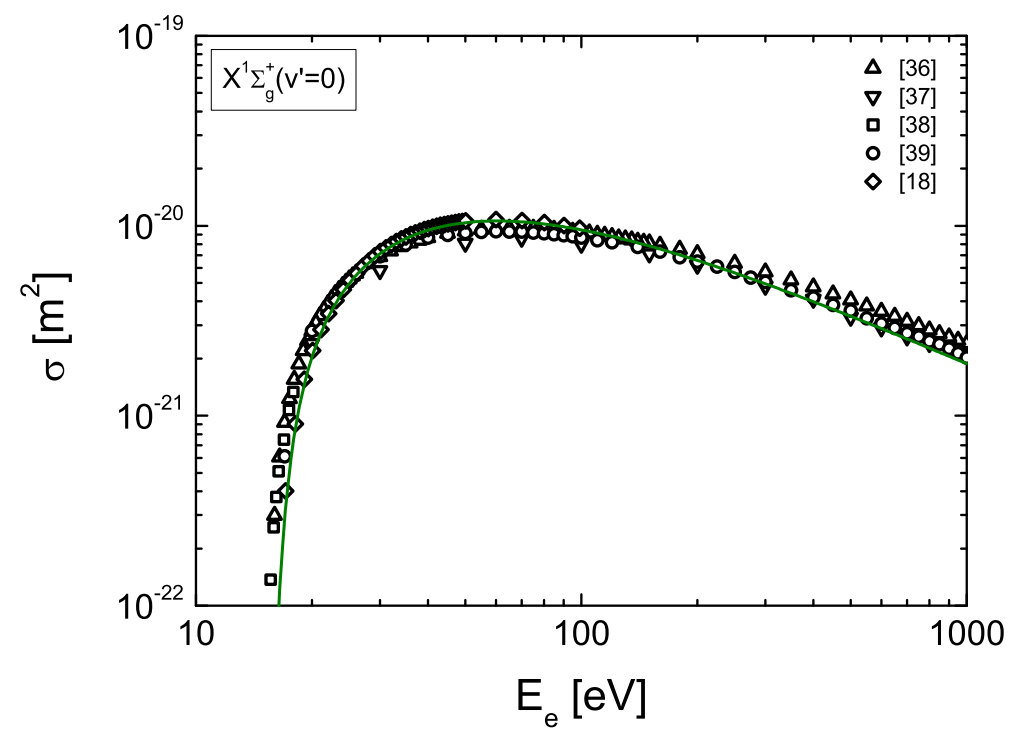

Figure 5: Cross sections for non-dissociative ionization of $\mathrm{v}=0$ in the molecular ground state. The continuous line represents the present results. The experimental and theoretical data available in the literature are represented by symbols.

[19] is based on the Gryzinski theory combined with the Franck-Condon theory and perfectly reproduces the present data.

Plotted in figure 6 is the present cross section, compared with data available from literature for the dissociative ionization, again for $v^{\prime}=0$ in the molecular ground state. Large deviations between the different cross sections are observed, attributed to the different measurement and calculation methods:

The experimental cross sections by [38] are available close to the reaction threshold only and have been measured by using an apparatus in which hydrogen gas particles are ionized by an electron gun. The two possible reaction channels for dissociative ionization (equations (2) and (3)) have been discriminated by tuning an electrostatic ion lens connected to a mass spectrometer. The absolute value of the cross sections have been determined by normalizing the sum of both channels to a previously measured total cross section [40].

The measurement method used in [39] is also based on an electron gun ionizing a target gas. A time-of-flight mass spectrometer is applied to discriminate between non-dissociative and dissociative ionization. The latter 


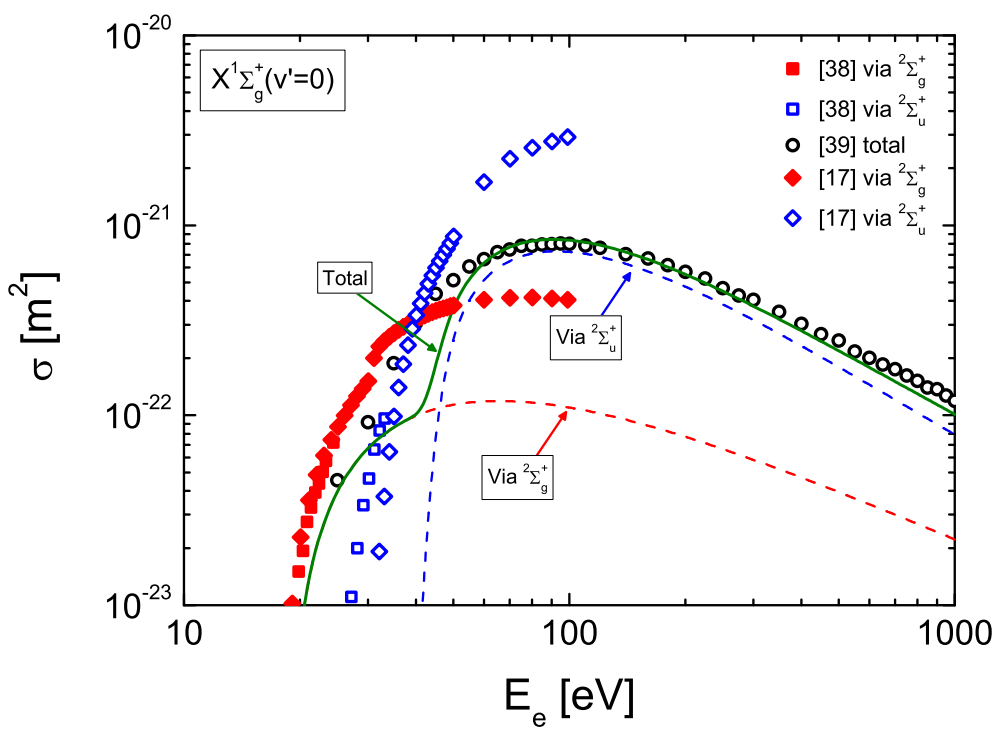

Figure 6: Cross sections for dissociative ionization of $\mathrm{v}=0$ in the molecular ground state. The continuous line represents the present total cross section, the dashed line the contributions of the two reaction channels to this total cross section. The experimental and theoretical data available in the literature are represented by symbols. 
channel is not resolved for its two possible reaction channels. The data from [39] can be considered as the most accurate experimental data available since absolute measurements have been performed: the density of atoms in the gas target, the number of impinging electrons and the number of created ions have been measured simultaneously. Hence, it was not necessary to normalize to some other data for obtaining the absolute value of the cross sections.

A very good agreement between the present data and the results of [39] is obtained for high electron energies $\left(E_{\mathrm{e}}>55 \mathrm{eV}\right)$ and close to the threshold energy $\left(E_{\mathrm{e}}<25 \mathrm{eV}\right)$. In the intermediate region the deviation increases to a maximum value of around three.

The calculated cross sections from [18] show a large and constant disagreement to the present data (factor $\approx 3.5$ ) and also to the experimentally determined cross sections by [39]. The data from [18] are based on the Gryzinski theory combined with the Franck-Condon theory. The observed discrepancy can be explained by the fact that — as mentioned already the $\delta$-function approximation has been applied instead of using numerically determined FCD and additionally the excitation channels of the dissociative ionization via the ionic state ${ }^{2} \Sigma_{g}^{+}$have not been considered properly.

The results shown in figures 5 and 6 prove that the applied framework reproduces the most accurate experimental cross sections available for nondissociative and dissociative ionization of $\mathrm{H}_{2}\left(X^{1} \Sigma_{g}^{+}, v^{\prime}=0\right)$. This finding is in general agreement with a statement made in [11]: the uncertainty of cross sections determined using the Gryzinski framework can be assumed to be smaller than a factor of two or three. Additionally, it was shown in [41] that the uncertainty can be significantly smaller for simply structured atoms or molecules. Hence, this quite simple classical framework was used for calculating state-to-state resolved calculations for all vibrational levels in the five electronic states $X^{1} \Sigma_{g}^{+}, E F^{1} \Sigma_{g}^{+}, B^{1} \Sigma_{u}^{+}, C^{1} \Pi_{u}, a^{3} \Sigma_{g}^{+}$and $c^{3} \Pi_{u}$ in the hydrogen molecule. The results of these calculations are shown in figures 7-12. The cross sections for non-dissociative ionization, plotted in the upper part of the figures, represent the sum for all upper vibrational levels $v^{\prime \prime}$ in the ground state ${ }^{2} \Sigma_{g}^{+}$of the ion. Due to strong differences in the dependence on the vibrational quantum number, the cross sections for both dissociative ionization channels are given separately, in the central and lower part of the figures, respectively. The scaling of the $\mathrm{y}$-axis was chosen in a way that in each case the maximum cross sections of the individual process are plotted and three orders of magnitude are covered. As a consequence, in some cases 
only four or less curves can be seen in the figures since the cross sections for the other vibrational states are significantly smaller than the data shown.

The cross sections are plotted for the first four vibrational levels $\left(v^{\prime}=\right.$ $0 \ldots 3)$, for $v^{\prime}=5$ and then for each third vibrational level up to the highest vibrational level in the specific electronic state or up to $v^{\prime}=20$ if more vibrational levels exist.

The dependence of the cross sections on the vibrational quantum number $v^{\prime}$ is strongly correlated to the corresponding Franck-Condon factors or Franck-Condon densities: it is particularly noticeable that with increasing quantum number $v^{\prime}$ the cross sections for non-dissociative ionization increase for the state $X^{1} \Sigma_{g}^{+}$, stay constant for $C^{1} \Pi_{u}$ and $c^{3} \Pi_{u}$ and show a indefinite behavior for the other states. The non-systematic dependence of the cross sections for the state $E F^{1} \Sigma_{g}^{+}$on $v^{\prime}$ is to be explained by the fact that the potential energy curve of this electronic state forms a double well. Some of the vibrational Eigenfunctions are in the left potential well, some in the right and for $v^{\prime} \geq 8$ the Eigenvalue is above the potential barrier between the two potential wells [22]. Hence, sudden changes in the FCF and FCD and the corresponding ionization cross sections can be observed from one vibrational quantum number to the next one.

For all considered electronic states in the molecule the cross sections for both dissociative ionization channels are roughly in the same order of magnitude. Exception is the state $C^{1} \Pi_{u}$. For this state the dissociative channel via the vibrational continuum of the ionic ground state ${ }^{2} \Sigma_{g}^{+}$is significantly less effective than the channel via the repulsive state ${ }^{2} \Sigma_{u}^{+}$. This finding is again directly connected to the vibrational structure of the FCD. Due to the relative position of the potential curves of $C^{1} \Pi_{u}$ and the ionic ground state ${ }^{2} \Sigma_{g}^{+}$, the closure relation (7) for the transition between these two states is fulfilled almost completely by the contribution of the FCF. The contribution of the FCD is almost negligible for all vibrational states $v^{\prime}$ and thus the absolute values of the dissociative ionization cross sections are also small.

\section{Conclusion}

The Gryzinski theory was applied in combination with the Franck-Condon theory to calculate vibrationally resolved ionization cross sections for the hydrogen molecule. As a prerequisite, Franck-Condon densities for nondissociative and dissociative ionization of the molecule have been calculated and verified. The applicability of the used theoretical framework was checked 
by comparing the present cross sections with cross sections available in the literature for $v^{\prime}=0$ in the molecular ground state. For non-dissociative ionization a very good agreement was found between the present data and experimentally determined as well as theoretical cross sections. While for dissociative ionization the agreement of the present data with the most reliable experimental results is also excellent, deviations within a factor of 3.5 are observed when comparing with theoretical cross sections from literature. These deviations are caused by a simplified theoretical framework which was applied for the previous calculations. Within this framework one of the reaction channels was neglected and simple approximation for the FranckCondon densities have been used.

A complete set of vibrationally resolved non-dissociative and dissociative ionization cross sections for the ground state and the first five electronically excited states in the hydrogen molecule has been calculated and is now available. These data constitute an important prerequisite for implementing ionizing and recombining processes into population models for atomic and molecular hydrogen. Such models based on the present data will be extremely useful for deepening the understanding of the relevant mechanisms in low temperature plasmas.

\section{Appendix A. Supplementary data}

A fitting procedure was applied to the cross sections for non-dissociative ionization and dissociative ionization of the ground state and the first five electronically excited states in the hydrogen molecule, $v^{\prime} \leq 20$.

\section{References}

[1] R. G. Isler, G. R. McKee, N. H. Brooks, W. P. West, et al., Phys. Plasmas 4 (1997) 2989.

[2] D. Lumma, J. L. Terry, B. Lipschultz, Phys. Plasmas 4 (1997) 2555.

[3] S. Krasheninnikov, A. Y. Pigarov, D. J. Sigmar, Phys. Lett. A 214 (1996) 285.

[4] A. Y. Pigarov, S. I. Krasheninnikov, Phys. Lett. A 222 (1996) 251.

[5] U. Fantz, D. Reiter, B. Heger, D. Coster, J. Nucl. Mater. 290 (2001) 367. 
[6] P. Franzen, H. D. Falter, U. Fantz, W. Kraus, et al., Nucl. Fusion 47 (2007) 264.

[7] U. Fantz, D. Reiter, B. Heger, D. Coster, Fusion Eng. Des. 66 (2001) 413.

[8] U. Fantz, D. Wünderlich, New J. Phys. 8 (2006) 301.

[9] J. J. A. V. den Dungen, O. Gabriel, H. S. M. M. E. D. C. Schram, R. Engeln, IEEE Transact. Plasma Sci. 36 (2008) 1028.

[10] W. E. N. van Harskamp, C. M. Brower, D. C. Schram, M. C. M. van de Sanden, et al., Phys. Rev. E 83 (2011) 036412.

[11] R. K. Janev, D. Reiter, U. Samm, Collision Proceses in LowTemperature Hydrogen Plasmas, Tech. Rep. JÜL-4105, Forschungszentrum Jülich, Jülich (2003).

[12] D. R. Bates, A. E. Kingston, R. W. P. McWirter, Proc. R. Soc. Lond. A 267 (1962) 297.

[13] T. Fujimoto, J. Phys. Soc. Japan 47 (1979) 265.

[14] R. W. P. McWirter, H. P. Summers, Phys Rev. A 12 (1975) 1872.

[15] T. E. Sharp, At. Data 2 (1971) 119.

[16] M. Parikh, Phys Rev. A 12 (1975) 1872.

[17] R. Celiberto, P. Cives, M. Cacciatore, M. Capitelli, et al., Chem. Phys. Lett. 169 (1990) 69.

[18] R. Celiberto, M. Capitelli, M. Cacciatore, Chem. Phys 140 (1990) 209.

[19] R. Celiberto, M. Capitelli, A. Laricchiuta, Phys. Script. T 96 (2002) 32.

[20] J. Franck, E. G. Dymond, Transactions of the Faraday Society 21 (1926) 536-542.

[21] E. Condon, Phys. Rev. 28 (1926) 1182-1201.

[22] U. Fantz, D. Wünderlich, At. Data Nucl. Data Tables 92 (2006) 853. 
[23] D. Wünderlich, U. Fantz, At. Data Nucl. Data Tables 97 (2011) 152.

[24] M. Gryzinski, Phys. Rev. 138 (1965) A336.

[25] E. Bauer, C. D. Bartky, J. Chem. Phys. 43 (1965) 2466.

[26] J. J. Thomson, Phil. Mag. 23 (1912) 449.

[27] L. Wolniewicz, J. Chem. Phys. 99 (1993) 1851.

[28] T. Orlikowski, G. Staszewska, L. Wolniewicz, Molec. Phys. 96 (1999) 1445.

[29] L. Wolniewicz, K. Dressler, J. Chem. Phys. 82 (1985) 3292.

[30] G. Staszewska, L. Wolniewicz, J. Molec. Spectr. 212 (2002) 208.

[31] L. Wolniewicz, G. Staszewska, J. Molec. Spectr. 220 (2003) 45.

[32] G. Staszewska, L. Wolniewicz, J. Molec. Spectr. 198 (1999) 416.

[33] W. Kolos, J. Rychlewski, J. Molec. Spectr. 169 (1995) 341.

[34] W. Kolos, J. Rychlewski, J. Molec. Spetr. 66 (1977) 428.

[35] D. M. Bishop, R. W. Wetmore, Mol. Phys. 26 (1973) 145.

[36] D. Rapp, P. Englander-Golden, J. Chem. Phys. 43 (1965) 1464.

[37] B. Adamcyk, A. J. H. Boerboom, B. L. Schram, J. Kistemaker, J. Chem. Phys. 44 (1966) 4640.

[38] A. Crowe, J. W. McConkey, J. Phys. B 6 (1973) 2088.

[39] H. C. Straub, P. Renault, B. G. Lindsay, K. A. Smith, et al., Phys. Rev. A 54 (1996) 2146.

[40] R. E. Golden, D. Rapp, Total cross sections for ionization of atoms and molecules by electron impact, Tech. Rep. 6-74-64-12, Lockheed Missiles and Space Company (1964).

[41] R. H. McFarland, Phys. Rev. 139 (1965) A40. 


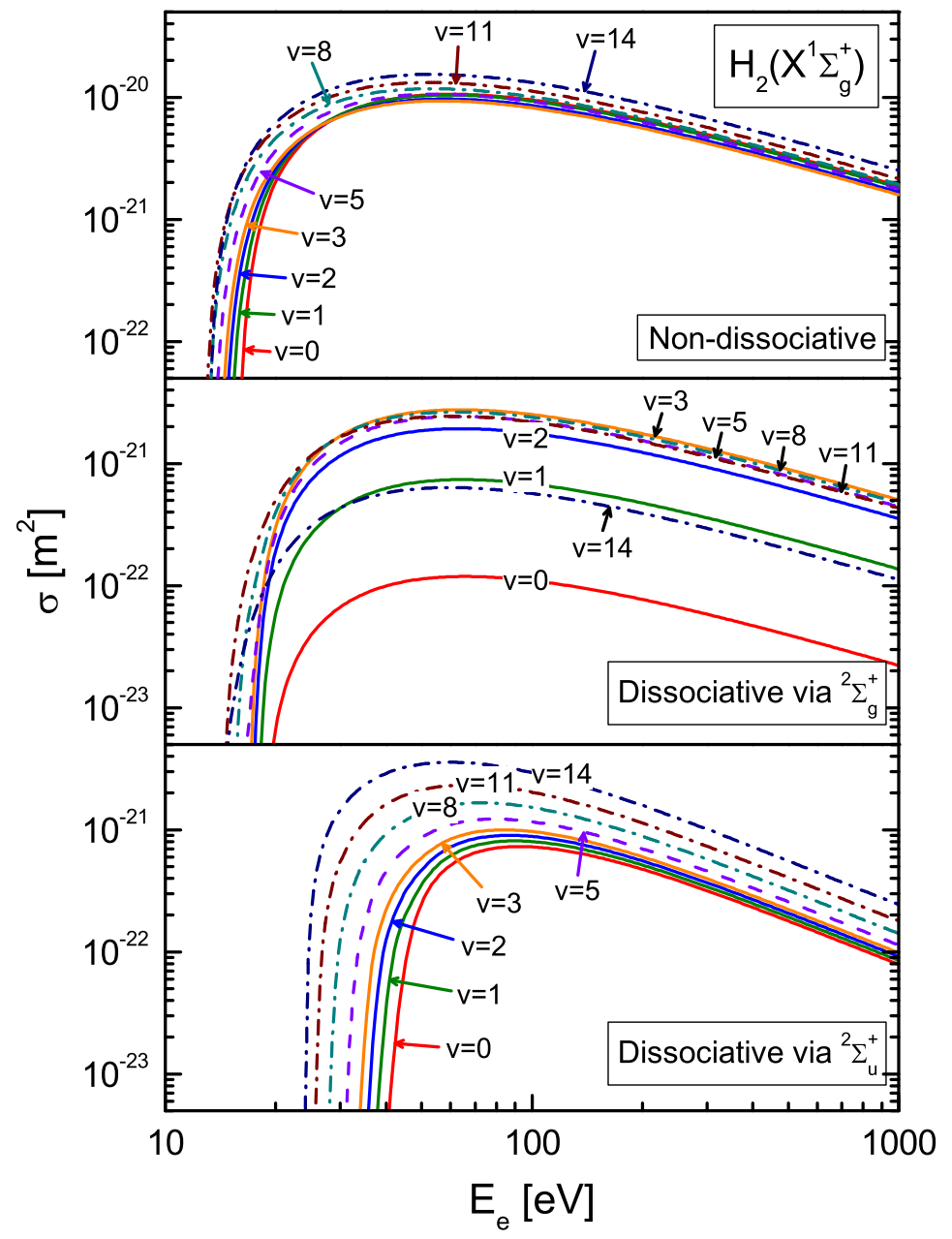

Figure 7: Calculated non-dissociative and dissociative ionization cross sections for the ground state $X^{1} \Sigma_{g}^{+}$of the hydrogen molecule. 


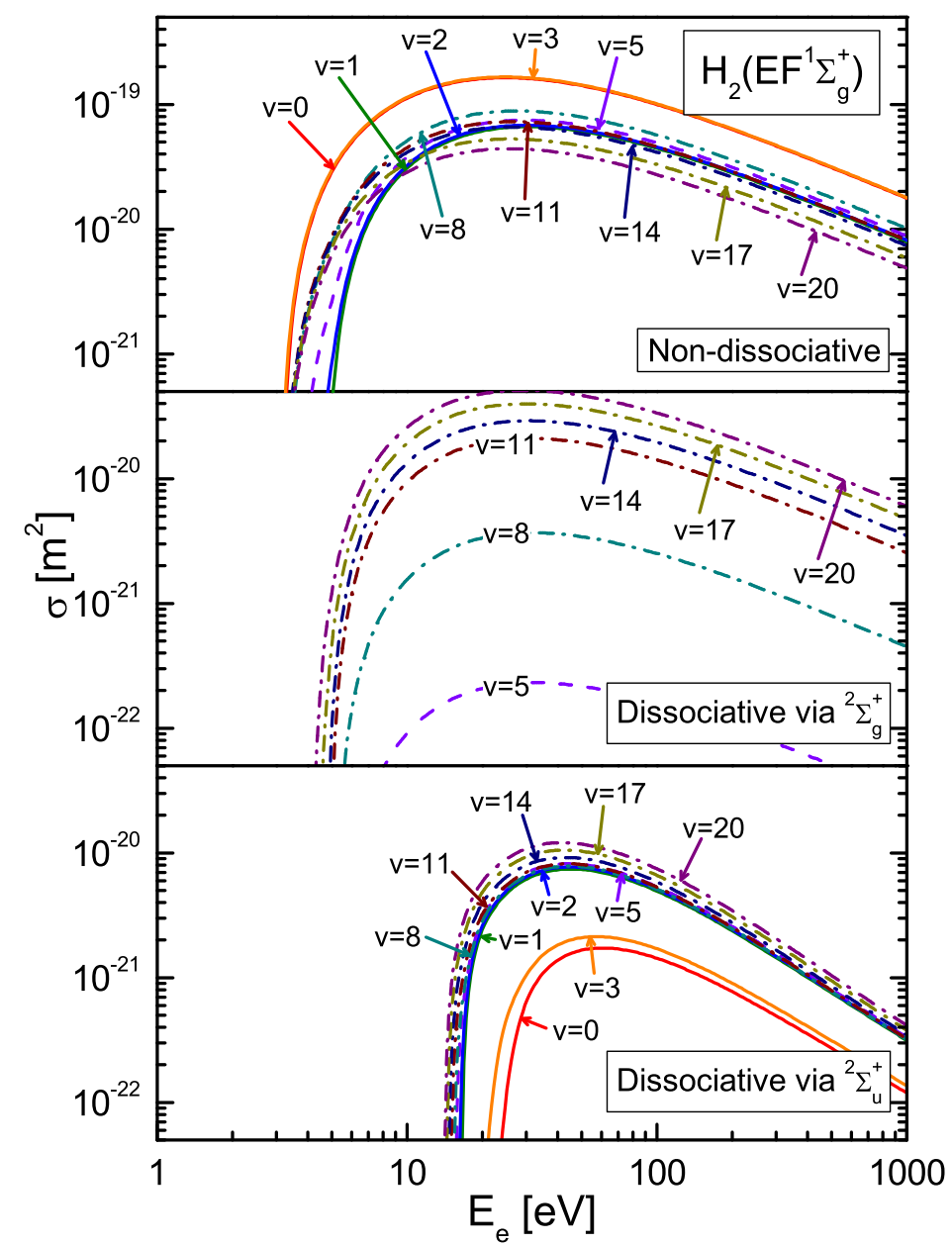

Figure 8: Calculated non-dissociative and dissociative ionization cross sections for the state $E F^{1} \Sigma_{g}^{+}$of the hydrogen molecule. 


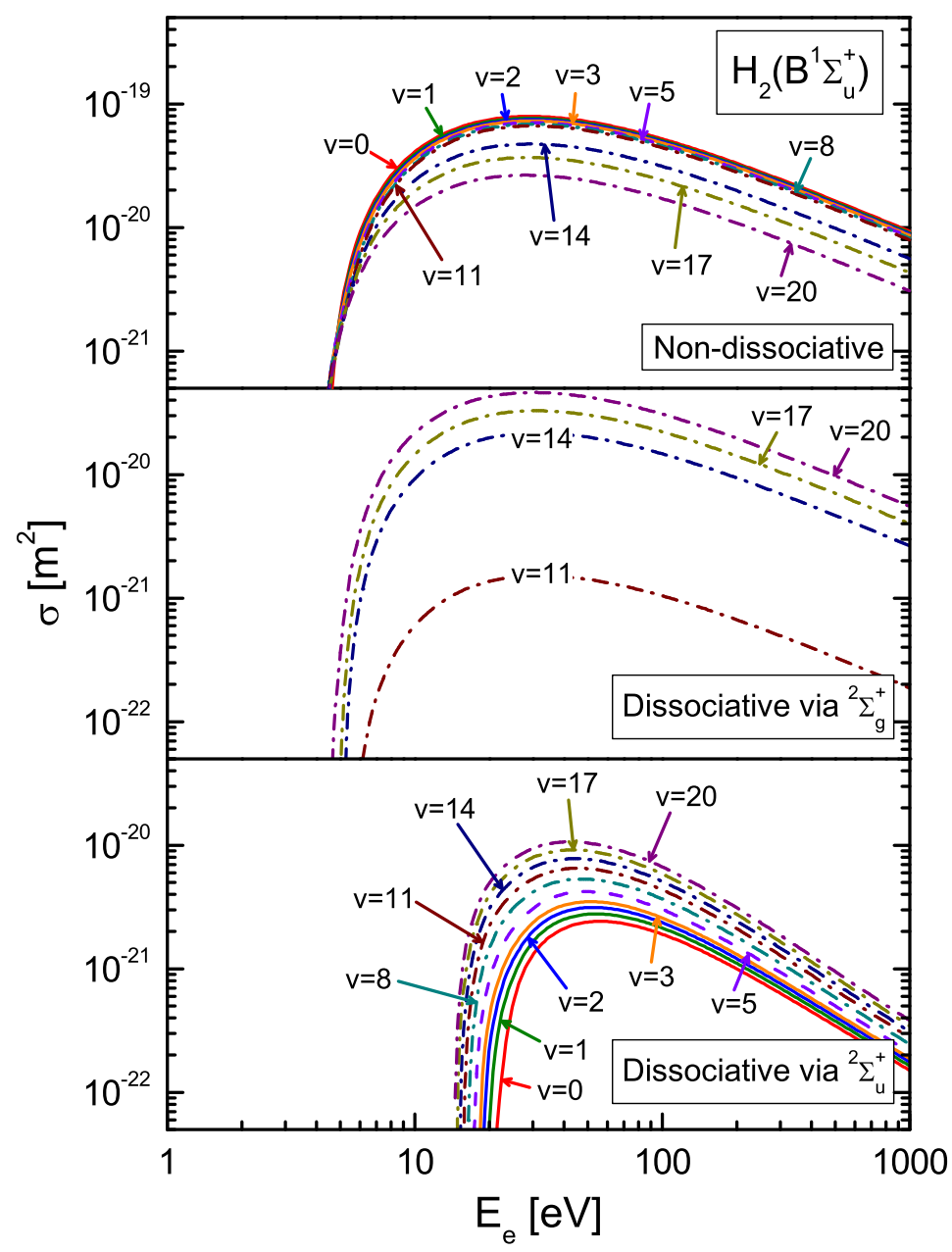

Figure 9: Calculated non-dissociative and dissociative ionization cross sections for the state $B^{1} \Sigma_{u}^{+}$of the hydrogen molecule. 


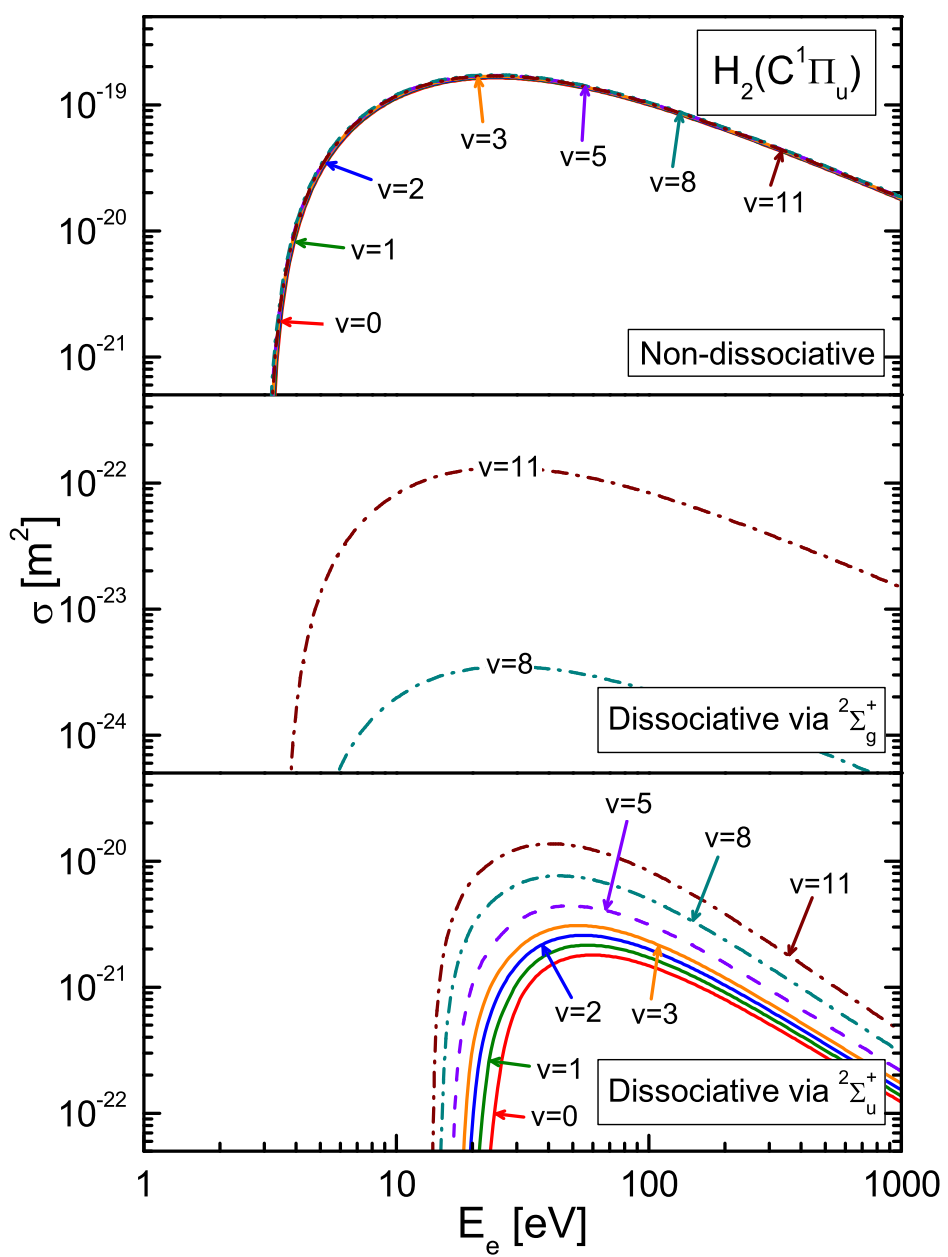

Figure 10: Calculated non-dissociative and dissociative ionization cross sections for the state $C^{1} \Pi_{u}$ of the hydrogen molecule. 


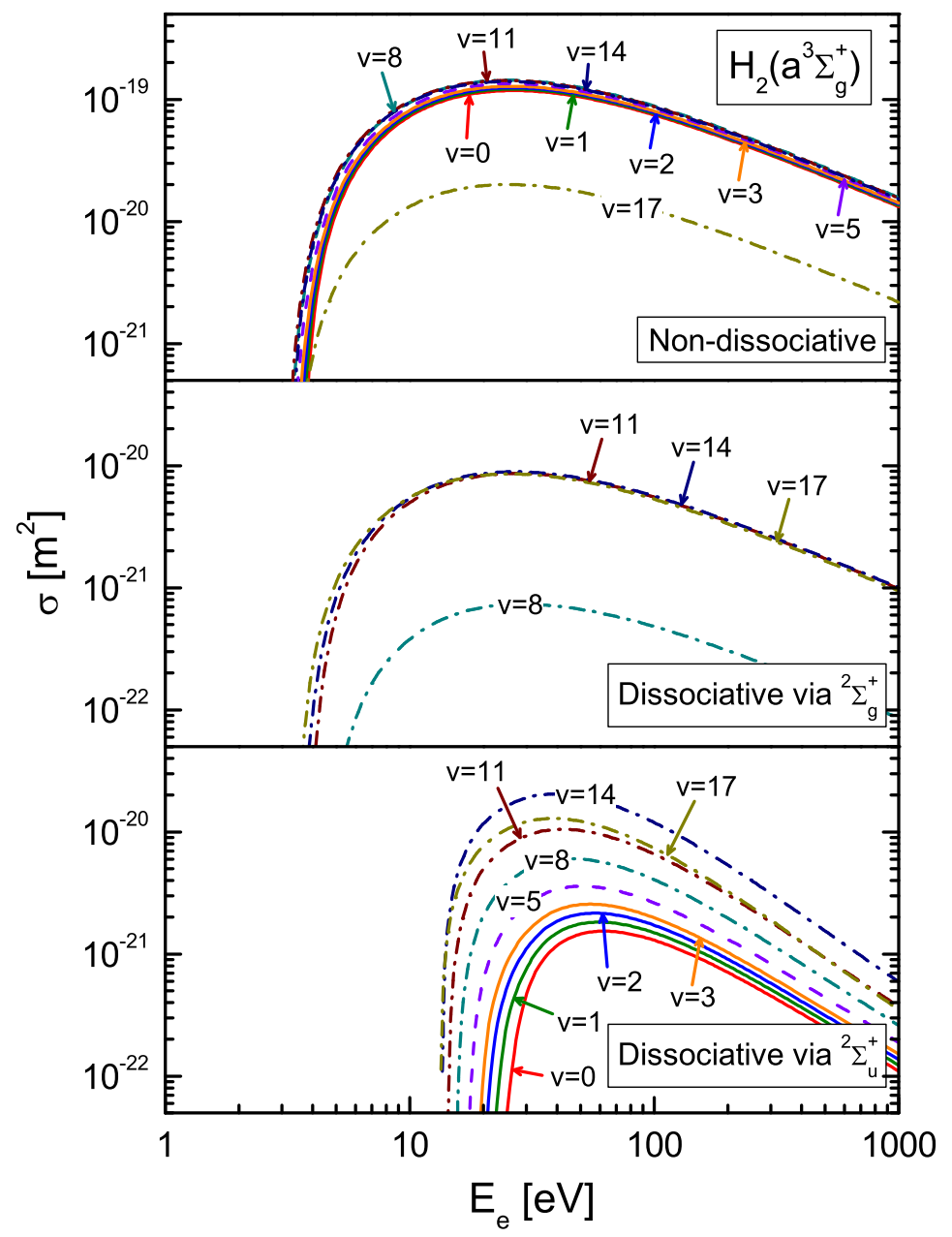

Figure 11: Calculated non-dissociative and dissociative ionization cross sections for the state $a^{3} \Sigma_{g}^{+}$of the hydrogen molecule. 


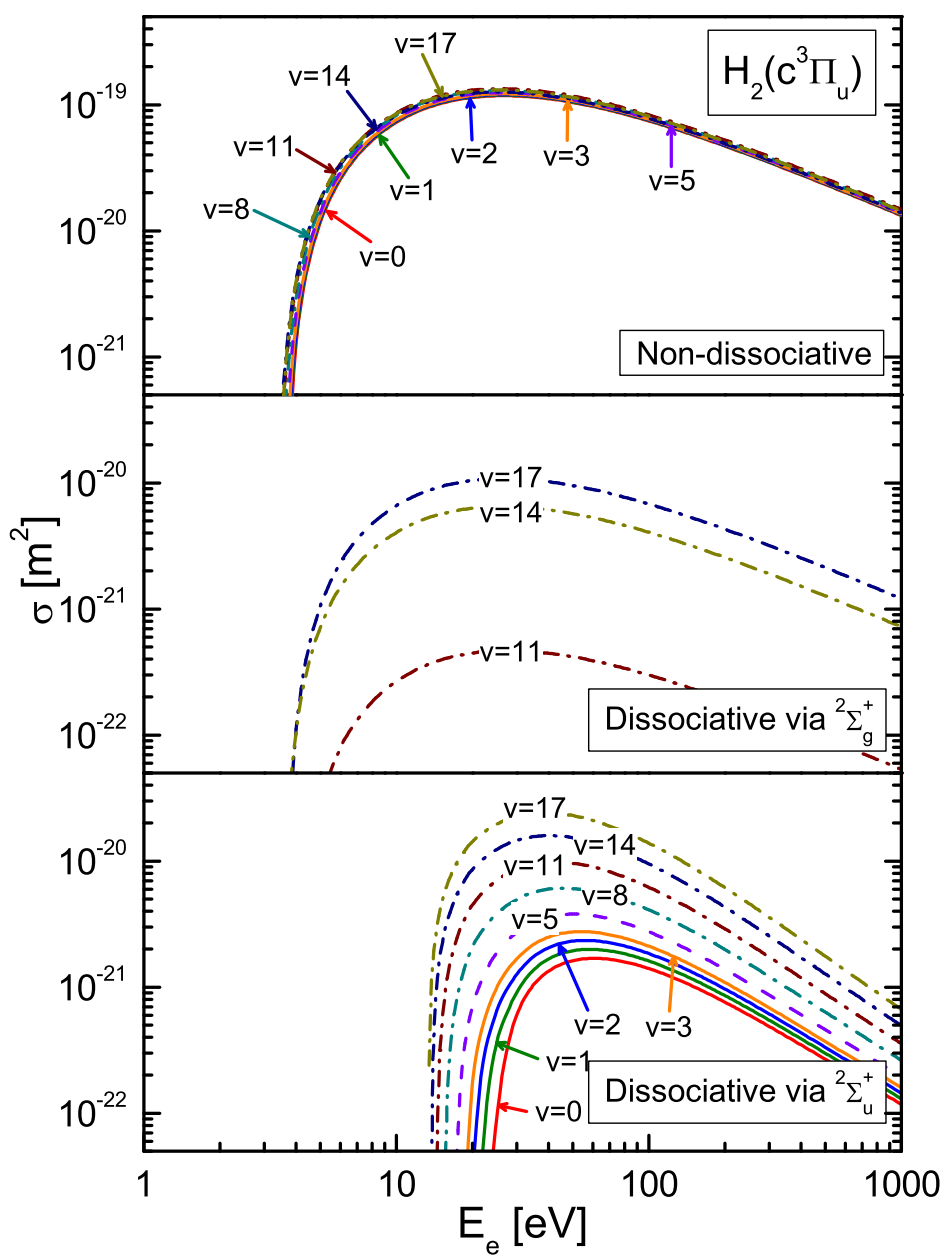

Figure 12: Calculated non-dissociative and dissociative ionization cross sections for the state $c^{3} \Pi_{u}$ of the hydrogen molecule. 